\title{
THE STUDY OF ARRHYTHMIAS FOLLOWING MYOCARDIAL INFARCTION OCCURING WITHIN ONE WEEK
}

\author{
Sarala. H. Tippannavar, Maharudra. S. Shekhanawar, N. Gunasheelan
}

1. Consultant Physician. Department of General Medicine, RTPS Hospital, Shaktinagar, Raichur.

2. Assistant Professor. Department of Biochemistry, Vijayanagar Institute of Medical Sciences.Bellary.

3. Professor, Department of General Medicine, Mysore Medical College \& Research Institute, Mysore

\section{CORRESPONDING AUTHOR}

Dr. Sarala. H. Tippannavar.

M.D. General Medicine, Consultant Physician,

RTPS Hospital, Shaktinagar 584170,

Raichur, Karnataka State.

E-mail: drsaralams@gmail.com,

Ph: $00919480899390,00919480899389$.

ABSTRACT: OBJECTIVES: Acute myocardial infarction continues to be a major health problem. $50 \%$ of deaths with acute myocardial infarction is said to occur within first 24 hours after myocardial infarction and is attributed to arrhythmias. Arrhythmic deaths remain the major cause of death with reduced left ventricular ejection fraction or frequent ventricular premature beats. The objective of this study is to assess various arrhythmias following myocardial infarction within one week and to study the association of these arrhythmias with left ventricular dysfunction. METHODS: Minimum of 100 cases diagnosed as acute myocardial infarction (European/ ACC Guidelines 2000) with arrhythmias collected by simple random sampling. Data was collected in a pre-tested proforma by meeting objectives of the study, detailed history, physical examination, ECG changes from day 1 to day 7 and whenever necessary, echocardiography to assess ejection fraction, and serum enzyme levels. RESULTS:

Male to female ratio with 4:1, mortality was more in the group with risk factors of hypertension, smoking and diabetes. Commonest arrhythmias noticed in this study were VPBs - 23\%, ST-22\%, BBB $-20 \%$, SB-19\%, VT $-6 \%$, and AF-4\%. In 17 out of 69 patients, thrombolysis therapy developed reperfusion arrhythmias of which VPBs being commonest. In addition to commonest arrhythmias there was a significant association between VT, AF and LV dysfunction. CONCLUSION: The commonest arrhythmias encountered were ventricular premature beats followed by sinus tachycardia, sinus bradycardia, bundle branch block and ventricular tachycardia. SB \& BBB were more common in IWMI where as ST, VPBs, AF and flutter were more common with AWMI. In addition to arrhythmias, LV dysfunction added to the mortality.

KEY WORDS: Myocardial infarction; Arrhythmias.

INTRODUCTION: There has been a substantial reduction in deaths from cardiovascular causes during the past 40-50 years, but cardiovascular disease remains the single most important cause of natural deaths in all developed countries of the world.

Deaths from arrhythmias in the setting of myocardial infarction (MI) have been one of the most frequent causes of sudden cardiac death. $60 \%$ of all deaths associated with acute myocardial infarction (AMI) occur within $1^{\text {st }}$ hour and are attributable to ventricular arrhythmias, in particular ventricular fibrillation ${ }^{1}$. Recent improvement in arrhythmia detection and treatment have had a major impact on the outcome of myocardial infarction. 
There is good correlation between the site of infarct and type of arrhythmias. Sinus bradycardia, sinoatrial, escape rhythms, complete heart block and Wenchebach type are usually associated with inferior wall myocardial infarction. Atrial and ventricular premature beats are more often seen in anterior wall myocardial infarction. ${ }^{2}$

There is a view that the cascade leading to sudden death from arrhythmias can be predicted by certain interactions among structural and functional abnormalities and search for new tools for prediction, the refinement of the existing tools, and the initiation of well designed intervention trials are the steps that must be taken towards the more efficient prevention of premature deaths from arrhythmias. ${ }^{3,4}$ Hence this study is undertaken to assess the commonest types of arrhythmias following myocardial infarction within one week and relation of these arrhythmias with site of infarction. Study is also undertaken to assess the association of most commonly occurred arrhythmias with LV (Left Ventricular) dysfunction.

METHODS: All patients diagnosed of acute myocardial infarction according to a consensus document of the Joint European Society of Cardiology/ American College of Cardiology Committee for the redefinition of myocardial infarction, guideline, 2000 with arrhythmias admitted to K.R. Hospital attached to Mysore Medical College and Research Institute, Mysore during period from $1^{\text {st }}$ January 2007 to $31^{\text {st }}$ July 2008 were recruited for the study. Each patient gave written, informed consent to participate in the study and the study protocol was approved by the institutional review board including ethical issues.

SAMPLE SIZE: Minimum of 100 cases diagnosed as acute myocardial infarction with arrhythmias were taken for the study by simple random sampling.

INCLUSION CRITERIA: Patients diagnosed as AMI as per - A consensus document of the Joint European Society of Cardiology/ American College of Cardiology Committee for the Redefinition of Myocardial Infarction.

CRITERIA FOR ACUTE MI, EVOLVING OR RECENT MYOCARDIAL INFARCTION: Typical rise and gradual fall (troponin) or more rapid rise and fall [CK(MB)] and biochemical markers of myocardial necrosis with one of the following.

Ischemic symptoms

Development of pathological Q waves

ECG changes indicative of ischemia (ST elevation or depression)

CRITERIA FOR ESTABLISHED MI: Development of new pathologic Q waves on serial ECGs.

EXCLUSION CRITERIA: Patients with,

(1) Previous history of documented arrhythmias

(2) Old MI

(3) History of MI more than one week.

Data was collected in a pre-tested proforma by meeting objectives of the study, detailed history, physical examination, thorough cardiovascular and other systemic examination and necessary investigations like ECG changes, echocardiography and serum enzyme levels. First ECG was taken immediately after admission. Patients were connected to cardiac monitor for a minimum of 48 hours and more if needed. Repeated ECGs were taken from day one to day seven whenever necessary and on occurrence of arrhythmias. 
All patients were evaluated for risk factors like diabetes mellitus, hypercholestrolemia, hypertension and smoking. Routine investigations were restricted to the patients who really needed them. Enzyme studies were done in most of the cases. Patients were kept in the ICCU for a period of five days and more in complicated cases.

RESULTS: A total of 100 patients with male and female ratio of 4:1, with AMI were included in the study. The maximum number of patients were found in the age group of $61-70$ years $(35 \%)$, followed by $51-60$ years (25\%), 41-50 years (19\%), 31-40 years $(10 \%), 71-80$ years $(7 \%), 21$ 30 years (3\%), and $81-90$ years (1\%).

In the study group, $48 \%$ of patients had history of hypertension, $38 \%$ of patients had smoking as a risk factor, $36 \%$ of patients had diabetes and $18 \%$ of patients had alcoholism as a risk factor.

The commonest symptom presented by patients was chest pain $95 \%$, followed by exertional breathlessness in $31 \%$, sweating in $16 \%$ of cases, vomiting in $8 \%$ of cases, $4 \%$ of cases palpitation and $2 \%$ of cases epigastric pain.

Most of patients (83\%) had ST segment elevation MI of which $34 \%$ had extensive anterior wall MI, 23\% had inferior wall MI, 14\% had anteroseptal wall MI, $6 \%$ had inferior wall with RV extension, $3 \%$ had lateral and anterolateral wall MI each, and non-ST elevation MI patients were $17 \%$.

The prevalence of various types of arrhythmias in acute myocardial infarction in relation to site of infarction is shown in table 1 . In arrhythmias of which ventricular arrhythmias were more common, $23 \%$ of cases had ventricular premature beats (VPBs), $6 \%$ of patients had ventricular tachycardia (VT), $2 \%$ of cases had ventricular fibrillation (VF) followed by sinus tachycardia (ST) in $22 \%$ of cases, $20 \%$ of patients had bundle branch block (BBB), $19 \%$ of patients had sinus bradycardia (SB), $4 \%$ had atrial fibrillation (AF), $2 \%$ had atrial flutter, $1 \%$ had couplets and nodal ectopics each.

In total of 22 cases, 8 cases of sinus tachycardia were seen in extensive anterior wall. In total of 19 cases, 12 cases of sinus bradycardia were seen in inferior wall MI. Total out of 23 cases, 13 cases of ventricular premature beats were seen in extensive anterior wall MI. Out of 100 cases, 38 cases of arrhythmias were seen in extensive anterior wall MI.

Prevalence of arrhythmias from day 1 to day 7 was studied. According to this most of the patients presented with symptoms after 1 day or 3 days. The presence of arrhythmia was taken as on day 1 or day 3, etc. According to the study, ventricular tachycardia, ventricular fibrillation and ventricular flutter occurred within 24 hours. VPBs occurred after 24-48 hours.

Sinus tachycardia and sinus bradycardia present on the first day at presentation of patient symptoms had reverted back to normal sinus rhythm. Bundle branch block occurred from day 1 to day 4 it was usually left bundle branch block (LBBB) or right bundle branch block (RBBB). Prevalence of arrhythmias is shown in table 2.

Table 3 shows $47.6 \%$ of inferior wall myocardial infarction patients had bundle branch block, followed by $23.8 \%$ of anteroseptal wall myocardial infarction patients had blocks and $19.04 \%$ of extensive anterior wall myocardial infarction had blocks. Total of $21 \%$ patients had one or the other type of atrioventricular blocks. The most commonest type of blocks encountered was LBBB (38.09\%) followed by $28.57 \%$ RBBB, $19.04 \%$ cases had complete heart block, $4.76 \%$ cases in bifascicular block, $\mathrm{III}^{0} \mathrm{AV}$ block and $\mathrm{II}^{0} \mathrm{AV}$ block respectively. 
Mortalities in reperfusion arrhythmias occurred of which ventricular premature beats were more common and were self terminated. One patient with VF after reperfusion expired after cardioversion. Details of reperfusion arrhythmias is shown in table 4.

Chi-square test was used to assess the association of arrhythmias in relation to left ventricular dysfunction (Table 5). Most of the patients with sinus tachycardia, atrial fibrillation, ventricular tachycardia and bundle branch block had less ejection fraction $(<40 \%)$, compared to patients with sinus bradycardia and VPBs (>40\%). The association of arrhythmias with left ventricular dysfunction was significant in patients with atrial fibrillation and ventricular tachycardias with ' $p$ ' value of 0.018 .

3 patients each with AF and VT had the least ejection fraction of 20-30\%, followed by patients with bundle branch block had EF of 31-40\%. Patients with VPBs and SB had near normal ejection fraction (EF) of 41-50\%, followed by patients with sinus tachycardia had an EF of $40 \%$.

DISCUSSION: The maximum incidence of acute myocardial infarction seen in this study was in the age group of $41-70$ years (79\%), of this $35 \%$ patients belong to $61-70$ years group. Only $3 \%$ of cases were below the age of 30 years. Age incidence in this study is almost similar to the studies done by Marthin TC et al. ${ }^{5}$ and Kakade SV et al. ${ }^{6}$ where $85 \%$ patients were between 35 and 75 years old. Age incidence is probably more common because of life style, economic status and multiple risk factors and life expectancy.

Incidence of acute myocardial infarction in this study was $80 \%$ in males which was more as compared to females (20\%). The study done by Kock HL et al. ${ }^{7}$ showed $72 \%$ male and $24 \%$ females. It is more common because of life style and more risk factors like hypertension, smoking, diabetes mellitus and alcohol.

In the present study, incidence of hypertension was $48 \%$ and incidence of smoking $38 \%$, $36 \%$ had diabetes mellitus and 18\% alcoholism. Mortality was more in the group with risk factors of hypertension, smoking and diabetes.

Sinus bradycardia was most commonly associated with inferior wall myocardial infarction. In the present study, 19 patients had sinus bradycardia, out of which 12 were of inferior wall MI. Similar observations were made by Michel Rotman et al. ${ }^{8}$ (10 - 30\%), Philip J Podrid, ${ }^{9}$ where $16 \%$ to $25 \%$ patients had sinus bradycardia particularly of inferior wall MI and posterior wall MI. It was most often transient.

In the present study, no deaths occurred in patients with sinus bradycardia and IWMI which is same as in the study done by Mall RR and Sayami A. ${ }^{10}$

In the present study, sinus tachycardia was present in $22 \%$ patients and commonly associated with anterior wall MI (9\% cases) compared to inferior wall MI (3\% cases). It represents an appropriate physiological response to left ventricular dysfunction, congestive heart failure (CHF) or stimulation and over activity of the sympathetic nervous system. This study compares well with the study done by Philip J Podrid. ${ }^{9}$

Atrial fibrillation ( $4 \%$ cases) and atrial flutter ( $2 \%$ cases) were seen in extensive anterior wall MI as most commonly in those who had significantly left ventricular dysfunction and CHF, and had increased mortality not because of arrhythmia itself, but to factors associated with it, particularly LV dysfunction and shock. Study done by Philip J Podrid ${ }^{9}$, Galcera Thomas J et al ${ }^{11}$ and Pizzetti $\mathrm{F}^{12}$ et al showed $\mathrm{AF}$ and flutter was associated with increased mortality in patients with LV dysfunction. 
Nodal ectopic was seen in $1 \%$ of cases and is similar to the study done by Galcera Thomas J et al. ${ }^{11}$ with $1 \%$ cases of nodal ectopic.

$20 \%$ of patients had bundle branch block. In the study done by Keith Newby et al. ${ }^{13}$ was $8-18 \%$ bundle branch block. In the present study, out of 20 cases, $8 \%$ cases seen were LBBB, $6 \%$ cases RBBB. The overall mortality rate was high compared to other patients in the present study. The study conducted by Keith Newby et al. ${ }^{13}$ shows high mortality.

Ventricular arrhythmias were seen in 31 cases of which VPBs in 23 cases, VT in 6 cases, VF in 2 cases. Study conducted by Julain Villacastin ${ }^{14}$ showed total incidence of VPB $12 \%$ and VT 18\% and Mossimo Zoni Berisso et al ${ }^{15}$ showed 19.7\% VPBs and VT 6.8\%.

In the present study, total incidence of reperfusion arrhythmias was 17 cases, out of which, VPBs in 9 cases, couplets 1 case, VF 1 case and VT 3 cases. In a study done by Mohamed Majidi et al. ${ }^{16}$ reperfusion arrhythmias in 157 patients were VF 4\% VPB 10\% and couplets $2 \%$.

In our study, one patient developed VF after reperfusion expired. In another study done by Ghuran $\mathrm{AV}^{17}$ and Lokas DRC et al ${ }^{18}$ VPBs are usually asymptomatic and their presence in the infarction period, regardless of frequency of complexity (bigeminy, trigeminy, etc.) has no relation to the mortality.

In the present study, about 60 patients showed varying ejection fraction in different types of arrhythmias. The ejection fraction found by echocardiography was least in AF and VT $(20-30 \%)$ compared to BBB and ST (40\%) and EF was better in sinus bradycardia (>40\%) showing less ventricular dysfunction over transient clinical outcome. There was also a significant association of arrhythmias with left ventricular dysfunction in patients with atrial fibrillation and ventricular tachycardias. This is in accordance with study conducted by Joaguin F Pombo ${ }^{19}$ and Pfisterer $\mathrm{M}^{20}$ in which 127 patients echocardiography reading shows incidence of severe ventricular arrhythmias was significantly higher with isolated right and left ventricular dysfunction compared with normal function. It was highest in VT and AF.

CONCLUSION: The commonest arrhythmias encountered were ventricular premature beats followed by sinus tachycardia, sinus bradycardia, bundle branch block and ventricular tachycardia. Most of the arrhythmias were seen in the first 48 hours. Mortality was more in the group with risk factors of hypertension, smoking and diabetes.

SB \& BBB were most commonly seen in IWMI where as ST, VPBs, AF and flutter were commonly seen in AWMI. ST, AF, flutter, VT and BBB were more commonly associated with LV dysfunction. There was a significant association of arrhythmias with LV dysfunction in patients with AF and VT. AF, BBB and flutter were associated with increased mortality in patients with LV dysfunction.

Further studies with larger sample size are needed to confirm the possible mechanisms between association of arrhythmias and LV dysfunction.

AKNOWLEDGEMENTS: We are grateful to Dr. D. Venkatesh, Director/ Dean, Mysore Medical College and Research Institute, Mysore for facilitating the study. We are also thankful to Dr. H. Vasudeva Naik, professor and HOD, Department of Medicine, Mysore Medical College and Research Institute, Mysore for his help in conducting the study. 


\section{REFERENCES:}

1. Podrid PJ. Ventricular arrhythmias after acute myocardial infarction, incidence and clinical features. BJMU; 2006 Apr 26.pp.1-8.

2. John KA. A history of cardiac arrhythmias. $2^{\text {nd }}$ ed. Chapter I. In: arrhythmias. WB Saunders Company;2000.

3. Hurikuri H., Castellanson A, Myerburg R,. Sudden detah due to cardiac arrhythmias. NEJM; 1990.

4. John MM, Zipes PD. Therapy for cardiac arrhythmias. $7^{\text {th }}$ ed. Chapter 30 In:

Heart disease -A textbook of cardiovascular medicine, Braunwald's. Pennsylvania:WB Saunders Company; 2001. P.713.

5. Martin TC, Longhuyzen HV. The age specific incidence of admission to te intensive care unit for acute myocardial infarction in Antigua and Barbuda. West Indian Med J 2007; 56(4):326-9.

6. Kakade SV, Tyagi NK, Kadam RN. Application of logistic regression to estimate prognosis in acute myocardial infarction. Ind J of Comm Med 2006; 31(2):338-45.

7. Kock HL, debruin A. Incidence of first acute myocardial infarction in the Netherlands. The Netherlands J Med 2007; 65(11):434-41.

8. Rotman M, Wanger G, Wallace A Brady arrhythmias in acute myocardial infarction. Circulation 1972; 45:703-22.

9. Podrid PJ. 0Arrthymias after acute myocardial infarction. Postgraduate Medicine 1997; 102(5):679-88.

10. Malla R, sayams A. Inhospital complications and mortality of patients of inferior wall myocardial infarction with right ventricular infarction. J Nepal Med Asso 2007;46(167):99-102.

11. Galecera TJ, Moreno M, Alberola G, Polo B, Aranaga M, Fernandez R, Incidence clinical characteristics and prognostic significance of supraventricular tacharrythmias in acute myocardial infarction.PMID;2007.

12. Pizzetti F, Turazza FM, Franzosi MG, Barlera S, Ledd A, Maggioni AP, et al. Incidence and prognostic significance of atrial fibrillation in acute myocardial i: the GISSI-3 data. Heart 2001; 86:527-32.

13. Newby K, Pisano E, Krucoff M, Green C, Natule A. Incidence and clinical relevance of the occurrence of Bundle- Branch Block in patients treated with thyombolytic therapy. Circulation 1996; 94:2424-8.

14. Villacastin J, Almendral J, Arenal A, Albertos J, Ormactxe J, Peinado R, et al. Incidence and clinical significance of multiple consecutive, appropriate, high energy discharges in patients with implanted cardiovester- Defibrillator. Circulation 1996; 93:753-62.

15. Berisso MZ, Daniele M. Value of programmed ventricular stimulation in predicting sudden death and sustained ventricular tachycardia in survivors of acute myocardial infarction. Am J Cardiol 1996; 77:673-80.

16. Majidi M, Kosinski A, Al-Khatib S, Lemmert M, Smolders L, weert A et al. Reperfusion ventricular arrhythmia burst in TIMI 3 flow restoration with primary angioplasty for anterior ST- elevation myocardial infarction: a more precise definition of reperfusion arrhythmias. Europace 2008; 10(8):988-97.

17. Ghuran AV, Camm AJ. Ischaemic heart disease presenting as arrhythmias. British Medical Bulletin 2011; 59:193-210. 
18. Lokas DRC, Connie R, Harrigue PS, Michael W. Familial sudden death is an important risk factor for primary ventricular fibrillation. A case control study in acute myocardial infarction patients. Circulation 2006; 114:1140-5.

19. Pombo J, Tray B, Russel R. Left Ventricular volumes and ejection fraction by echocardiography. Circulation 1971; 43:480.

20. Pfisterer M, Emmengegges H, Soler M, Burkart F, Prognostic significance of right ventricular ejection fraction from persistent complex ventricular arrhythmias and / or sudden cardiac death after first myocardial infarction: relation to infract location, size and left ventricular function. Eur Heart J 1986; 7(4):289-98.

Table 1 : Types of arrhythmias in relation to site of infarction

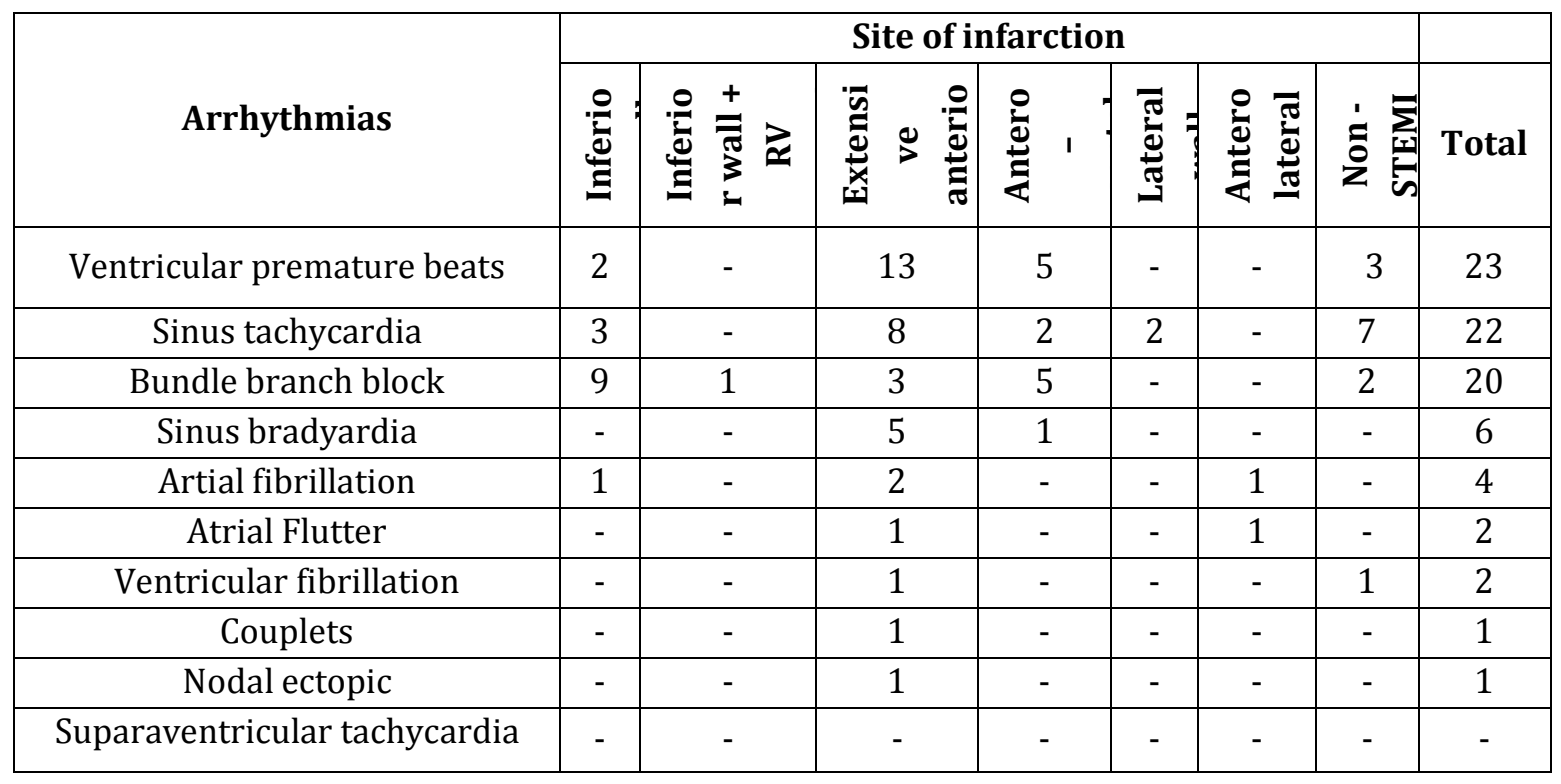

Table 2: Types of arrhythmias from day 1 to day 7

\begin{tabular}{|c|c|c|c|c|c|c|c|}
\hline Arrhythmias & $\begin{array}{l}\vec{\lambda} \\
\overrightarrow{0}\end{array}$ & $\begin{array}{l}N \\
\vec{\sigma} \\
\overrightarrow{0}\end{array}$ & $\begin{array}{l}n \\
\stackrel{\tilde{D}}{0}\end{array}$ & $\begin{array}{l}+ \\
\vec{\sigma} \\
\overrightarrow{0}\end{array}$ & $\begin{array}{l}10 \\
\text { 己̊ }\end{array}$ & $\begin{array}{l}0 \\
\overrightarrow{0} \\
0\end{array}$ & $\hat{\overrightarrow{0}}$ \\
\hline Ventricular premature beats & - & 7 & 7 & 5 & 1 & 1 & 2 \\
\hline Sinus tachycardia & 7 & 6 & 6 & 2 & 1 & - & - \\
\hline Bundle branch block & 5 & 6 & 6 & 3 & - & - & - \\
\hline Sinus bardycardia & 9 & 9 & 1 & - & - & - & - \\
\hline Ventricular tachycardia & 6 & - & - & - & - & - & - \\
\hline Atrial fibrillation & 4 & - & - & - & - & - & - \\
\hline Atrial flutter & 2 & - & - & - & - & - & - \\
\hline Ventricular fibrillation & 2 & - & - & - & - & - & - \\
\hline Couplets & - & 1 & - & - & - & - & - \\
\hline Nodal ectopic & - & 1 & - & - & - & - & - \\
\hline Others & - & - & - & - & - & - & - \\
\hline Total & 35 & 30 & 20 & 10 & 2 & 1 & 2 \\
\hline
\end{tabular}


Table 3: Types of blocks in relation to site of infraction

\begin{tabular}{|c|c|c|c|c|c|c|c|c|}
\hline \multirow[b]{2}{*}{ Site of infarction } & \multicolumn{6}{|c|}{ Type of block } & \multicolumn{2}{|c|}{ Total } \\
\hline & 黄 & 魚 & 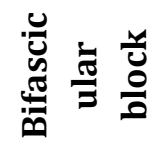 & 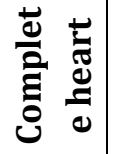 & $\geqq \frac{y}{3}$ & $\gtreqless \frac{y}{\circlearrowright}$ & $\dot{0}^{\circ}$ & $\%$ \\
\hline Inferior wall & 2 & 5 & - & 2 & - & 1 & 10 & $47.6 \%$ \\
\hline Anteroseptal & 1 & 2 & - & 2 & - & - & 5 & $23.8 \%$ \\
\hline Extensive anterior wall & 2 & 1 & 1 & - & 1 & - & 4 & $19.04 \%$ \\
\hline $\begin{array}{c}\text { Inferior wall + RV } \\
\text { extension }\end{array}$ & - & - & - & - & - & - & 1 & $4.76 \%$ \\
\hline Non - STEMI & 1 & - & - & - & - & - & 1 & $4.76 \%$ \\
\hline Lateral & - & - & - & - & - & - & - & - \\
\hline Anterolateral & - & - & - & - & - & - & - & - \\
\hline \multicolumn{7}{|c|}{ Total } & 21 & \\
\hline
\end{tabular}

Table 4: Reperfusion Arrhythmias

\begin{tabular}{|l|c|c|c|}
\hline \multicolumn{1}{|c|}{ Type } & Number of cases & Self termination & Mortality \\
\hline Occasional VPBs & 7 & 7 & - \\
\hline Multiple VPBs & 2 & 2 & - \\
\hline Couplets & 1 & - & 1 \\
\hline Atrial flutter & 1 & - & 1 \\
\hline Ventricular tachycardia & 3 & 1 & - \\
\hline Venricular bigeminy & 1 & 1 & 1 \\
\hline Ventricular fibrillation & 1 & - & - \\
\hline AV dissociation & 1 & 1 & \\
\hline
\end{tabular}


Table 5: Association of arrhythmias with left ventricular dysfunction

\begin{tabular}{|c|c|c|c|}
\hline \multirow{2}{*}{ Types of arrythmias } & \multicolumn{2}{|c|}{ Ejection fraction } & \multirow{2}{*}{ P Value } \\
\cline { 2 - 3 } & $<\mathbf{4 0 \%}$ & $\mathbf{> 4 0 \%}$ & \\
\hline Sinus tachycardia & $10(62.5 \%)$ & $6(37.5 \%)$ & \\
\hline Sinus bardycardia & $2(20 \%)$ & $8(80 \%)$ & \\
\hline Atrial fibrillation & $3(100 \%)$ & $0(0 \%)$ & \multirow{2}{*}{$\mathbf{0 . 0 1 8}$} \\
\hline VPBs & $7(41.2 \%)$ & $10(58.8 \%)$ & \\
\hline Ventricular tachycardia & $3(100 \%)$ & $0(0 \%)$ & \\
\hline Bundle branch block & $8(80 \%)$ & $2(20 \%)$ & \\
\hline Nodal ectopic & $0(0 \%)$ & $1(100 \%)$ & \\
\hline Total & $\mathbf{3 3}$ & $\mathbf{2 7}$ & \\
\hline
\end{tabular}

Chi-square value: 15.28, Degree of freedom: 6.

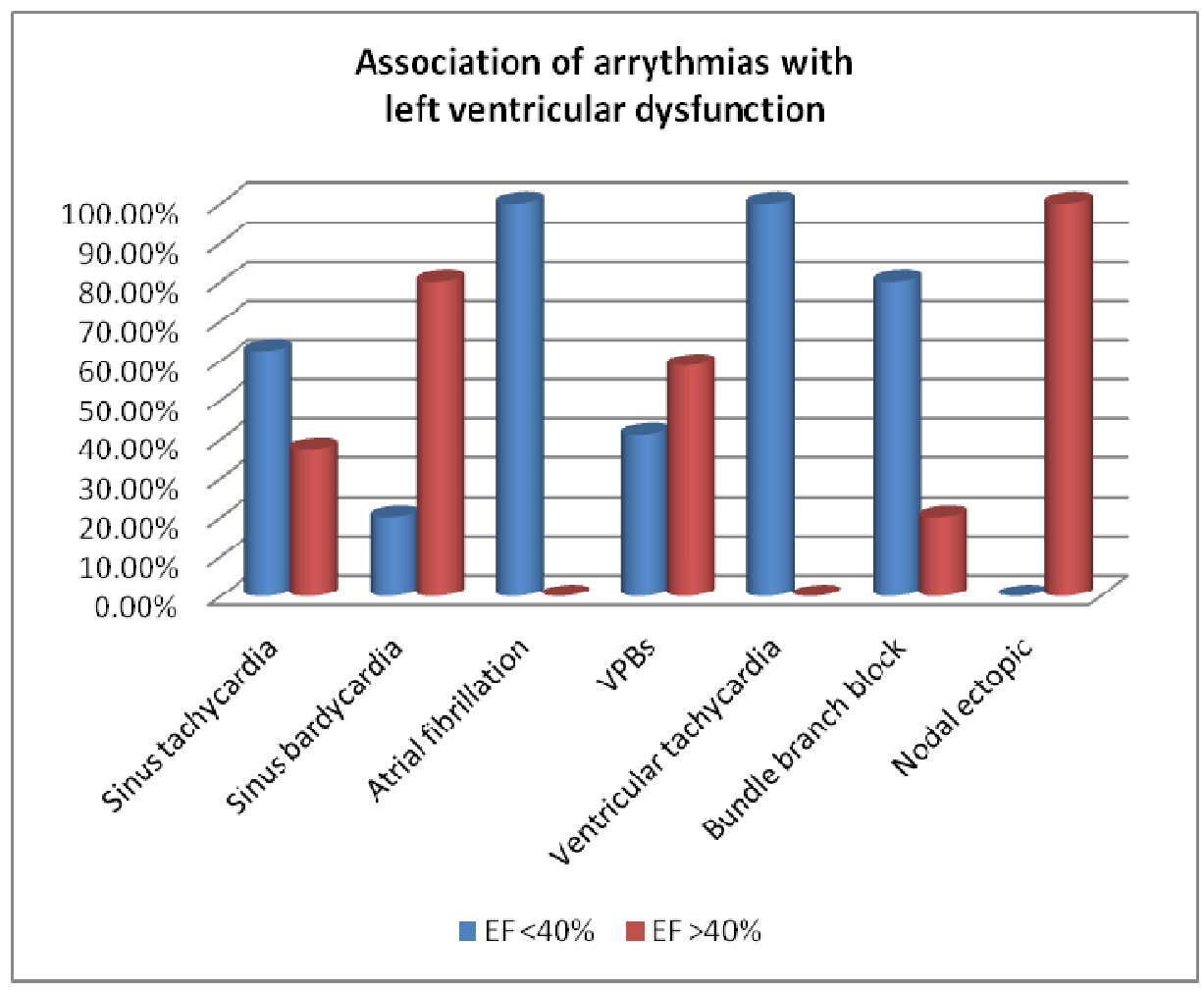

